Mathematical Modelling and AnAlysis

Volume 20 Number 2, March 2015, 168-187

http://dx.doi.org/10.3846/13926292.2015.1020455

(c) Vilnius Gediminas Technical University, 2015
Publisher: Taylor\&Francis and VGTU

http://www.tandfonline.com/TMMA

ISSN: $1392-6292$

eISSN: $1648-3510$

\title{
Traveling Wave Solutions in a Stage-Structured Delayed Reaction-Diffusion Model with Advection
}

\author{
Liang Zhang ${ }^{a, b}$ and Huiyan Zhao $^{b}$ \\ ${ }^{a}$ Institute of Applied Mathematics, College of science, Northwest A\&F \\ University
}

No. 22 Xinong Road, Yangling, 712100 Shaanxi, China

${ }^{b}$ State Key Laboratory of Crop Stress Biology in Arid Areas, School of Plant

Protection, Northwest A\&F University

No. 22 Xinong Road, Yangling, 712100 Shaanxi, China

E-mail: zhanglsd@126.com

E-mail(corresp.): zhaohy@nwsuaf.edu.cn

Received May 11, 2014; revised January 15, 2015; published online March 15, 2015

\begin{abstract}
We investigate a stage-structured delayed reaction-diffusion model with advection that describes competition between two mature species in water flow. Time delays are incorporated to measure the time lengths from birth to maturity of the populations. We show there exists a finite positive number $c^{*}$ that can be characterized as the slowest spreading speed of traveling wave solutions connecting two mono-culture equilibria or connecting a mono-culture with the coexistence equilibrium. The model and mathematical result in [J.F.M. Al-Omari, S.A. Gourley, Stability and travelling fronts in Lotka-Volterra competition models with stage structure, SIAM J. Appl. Math. 63 (2003) 2063-2086] are generalized.
\end{abstract}

Keywords: competition, stage structure, time delay, advection, travelling wave solutions.

AMS Subject Classification: 92D25; 34D23.

\section{Introduction}

In 1990, Aiello and Freedman [1] developed the following time-delay model of single-species growth with stage structure:

$$
\begin{aligned}
& u_{i, t}=\alpha u_{m}(t)-\gamma u_{i}(t)-\alpha e^{-\gamma \tau} u_{m}(t-\tau), \\
& u_{m, t}=\alpha e^{-\gamma \tau} u_{m}(t-\tau)-\beta u_{m}^{2}
\end{aligned}
$$

where $\alpha, \beta, \gamma$ and $\tau$ are positive constants. In this model, $u_{i}$ and $u_{m}$ denote, respectively, the numbers of immature and mature members of the population. The parameter $\tau$ in model measures the time from birth to maturity. The 
rate at which individuals are born is taken to be proportional to the number of matures at that time; this is the $\alpha u_{m}(t)$ term. And $e^{-\gamma \tau}$ terms allow for the fact that not all immatures survive to maturity $(\gamma$ is the death rate of immatures, while $\beta$ measures deaths of matures).

When the individuals are allowed for moving around, in 2003 Gourley and Kuang [5] initially studied the diffusive version of the system (1.1):

$$
\begin{aligned}
u_{i, t} & =d_{i} u_{i, x x}+\alpha u_{m}-\gamma u_{i}-\alpha e^{-\gamma \tau} \int_{\mathbb{R}} \frac{1}{\sqrt{4 \pi d_{i} \tau}} e^{\frac{-(x-y)^{2}}{4 d_{i} \tau}} u_{m}(t-\tau, y) d y, \\
u_{m, t} & =d_{m} u_{m, x x}+\alpha e^{-\gamma \tau} \int_{\mathbb{R}} \frac{1}{\sqrt{4 \pi d_{i} \tau}} e^{\frac{-(x-y)^{2}}{4 d_{i} \tau}} u_{m}(t-\tau, y) d y-\beta u_{m}^{2},
\end{aligned}
$$

where $d_{i}>0\left(d_{m}>0\right)$ is the diffusivity of the immature (mature) species. If the immatures are not moving, then we would allow $d_{i} \rightarrow 0$ in

$$
\int_{\mathbb{R}} \frac{1}{\sqrt{4 \pi d_{i} \tau}} e^{\frac{-(x-y)^{2}}{4 d_{i} \tau}} \times e^{-\gamma \tau} \alpha u_{m}(t-\tau, y) d y
$$

to conclude that the corresponding expression is $e^{-\gamma \tau} \alpha u_{m}(t-\tau, x)$. If $d_{i}>0$, expression (1.3) total up the individuals of population $u$ born at time $t-\tau$ in all parts of the domain that are still alive at time $t$ and have just reached maturity and arrived at $x$.

It is well known that competition both within and between species is an important topic in ecology, especially community ecology. Observe that system (1.2) is not a fully coupled system in that the second equation, for the mature population $u_{m}$, can be solved independently of the first. Consideration of this second equation alone is an interesting and non-trivial mathematical problem in its own way. Therefore Al-Omari and Gourley [2] studied the following Lotka-Volterra competition model:

$$
\begin{aligned}
& U_{1, t}=d_{1} U_{1, x x}+\alpha_{1} e^{-\gamma_{1} \tau_{1}} U_{1}\left(t-\tau_{1}, x\right)-\beta_{1} U_{1}^{2}(t, x)-c_{1} U_{1}(t, x) U_{2}(t, x), \\
& U_{2, t}=d_{2} U_{2, x x}+\alpha_{2} e^{-\gamma_{2} \tau_{2}} U_{2}\left(t-\tau_{2}, x\right)-\beta_{2} U_{2}^{2}(t, x)-c_{2} U_{1}(t, x) U_{2}(t, x) .
\end{aligned}
$$

In model (1.4), it is assumed that competition occurs only between the adults, since many species strongly protect their young, and the immature population are not moving.

In the real world, there are many cases that species live in the media where the diffusion moves to a certain direction. Typical examples include aquatic organisms in stream, and marine organisms with larval dispersal influenced by ocean currents. When one direction in the random walk is favored, an equation with a first order derivative is the result:

$$
u_{, t}=d u_{, x x}-v u_{, x}+R(u)
$$

The first order derivative term on the right hand side of the equation (1.5) is called an advection term, and the equation (1.5) is called a reaction-diffusion equation with advection.

When the mature individual movement is described as a combination of advection corresponding to the one-dimensional medium with a undirectional 
Table 1. Parameter description for model (1.6).

\begin{tabular}{ll}
\hline Parameter & Description of parameter \\
\hline$U_{i}(t, x)$ & population density of immature members $U$ at time $t$ and point $x$ \\
$U_{m}(t, x)$ & population density of mature members $U$ at time $t$ and point $x$ \\
$V_{i}(t, x)$ & population density of immature members $V$ at time $t$ and point $x$ \\
$V_{m}(t, x)$ & population density of mature members $V$ at time $t$ and point $x$ \\
$d_{U_{m}}$ & diffusion coefficient of mature population $U$ \\
$d_{V_{m}}$ & diffusion coefficient of mature population $V$ \\
$a_{U_{m}}$ & advection speed of mature population $U$ experienced by the organism \\
$a_{V_{m}}$ & advection speed of mature population $V$ experienced by the organism \\
$\alpha_{1}$ & birth rate of population $U$ \\
$\alpha_{2}$ & birth rate of population $V$ \\
$\gamma_{1}$ & death rate of immature population $U$ \\
$\gamma_{2}$ & death rate of immature population $V$ \\
$\beta_{1}$ & death rate of mature population $U$ \\
$\beta_{2}$ & death rate of mature population $V$ \\
$\tau_{1}$ & time delay from birth to maturity of population $U$ \\
$\tau_{2}$ & time delay from birth to maturity of population $V$ \\
$c_{1}$ & competitive effect of the species $V$ has on the mature of species $U$ \\
$c_{2}$ & competitive effect of the species $U$ has on the mature of species $V$ \\
\hline
\end{tabular}

flow as experienced by the organisms and diffusion corresponding to the heterogeneous stream flow and individual swimming, we are concerned with the following delayed competition reaction-diffusion model with stage structure and advection:

$$
\begin{aligned}
& U_{i, t}=\alpha_{1} U_{m}-\gamma_{1} U_{i}-\alpha_{1} e^{-\gamma_{1} \tau_{1}} U_{m}\left(t-\tau_{1}, x\right) \\
& U_{m, t}=d_{U_{m}} U_{m, x x}-a_{U_{m}} U_{m, x}+\alpha_{1} e^{-\gamma_{1} \tau_{1}} U_{m}\left(t-\tau_{1}, x\right)-\beta_{1} U_{m}^{2}-c_{1} U_{m} V_{m}, \\
& V_{i, t}=\alpha_{2} V_{m}-\gamma_{2} V_{i}-\alpha_{2} e^{-\gamma_{2} \tau_{2}} V_{m}\left(t-\tau_{2}, x\right) \\
& V_{m, t}=d_{V_{m}} V_{m, x x}-a_{V_{m}} V_{m, x}+\alpha_{2} e^{-\gamma_{2} \tau_{2}} V_{m}\left(t-\tau_{2}, x\right)-\beta_{2} V_{m}^{2}-c_{2} U_{m} V_{m}
\end{aligned}
$$

Here, we assume the immature individuals always stay on the stationary until they grow to maturity during a period of time, only the mature individuals drift in the water flow, and competition occurs only between the adults. A descriptions of all parameters can be found in Table 1 and all $c$-values are positive. In the model (1.6) it is assumed that competition effects are of the classical LotkaVolterra kind, while death of the matures for each population is modelled by quadratic terms, as in the logistic equation.

Since the second and the fourth equations in system (1.6) are uncoupled from the first and the third equations, it is sufficient to consider a simplified model with the first and third equations merged:

$$
\begin{aligned}
& u_{, t}=d_{1} u_{, x x}-a_{1} u_{, x}+\alpha_{1} u\left(t-\tau_{1}, x\right)-\eta_{1} u^{2}-p_{1} u v, \\
& v_{, t}=d_{2} v_{, x x}-a_{2} v_{, x}+\alpha_{2} v\left(t-\tau_{2}, x\right)-\eta_{2} v^{2}-p_{2} u v .
\end{aligned}
$$

Here $u(t, x)$ and $v(t, x)$ represent densities of adult members of two species $u$ and $v$ at time $t$ and point $x$, respectively. $d_{1}>0\left(d_{2}>0\right)$ is the diffusion coefficient of the adult population $u(v) . a_{1}>0\left(a_{2}>0\right)$ is the advection speed experienced by the organism. $\alpha_{1}\left(\alpha_{2}\right)$ combining two factors: the per 
capita birth rate and the survival rate of immature for the population $u(v)$ during the immature stage.

Mathematical modelling has been central to the development of general invasion theory (e.g., $[12,13,14,15,16]$ ). System in the form of reaction-diffusion equations and integro-difference equations are commonly used to describe biological invasion process. In ecology, the existence of travelling wave solutions connecting two equilibria means that the unstable equilibrium is taken over by the stable one in space as time increases. Studies on existence of traveling waves in such system have received considerable attention, and many noteworthy findings have come out of this field, e.g., [2,3, 5, 6, 7, 8, 9, 10,17, 18, 19, 20, 21,22].

Al-Omari and Gourley [2] studied the existence of traveling wave solutions connecting two mono-culture equilibria in model (1.4) under a technical assumption by using the theory of monotone iteration scheme developed by $\mathrm{Wu}$ and Zou [20]. This presents the invasion by the strong species of territory previously inhabited only by the weaker. The results in [2] made significant progress in establishing travelling wave solutions for model (1.4). It is pointed out in [2] that the technical assumption for the existence of traveling wave solutions may not be essential. In this paper, we attempt to provide the sharpest result regarding the existence of travelling wave solutions connecting an unstable mono-culture equilibrium with a nontrivial equilibrium in model (1.7) using our abstract result for general delayed recursion model established in [7]. We show that there exists a finite positive number $c^{*}$ that can be characterized as the slowest speed of travelling wave solutions connecting two mono-culture equilibria or connecting a mono-culture with the coexistence equilibrium.

The rest of this paper is organized as follows: In Section 2, we present some preliminaries including equilibria and stability for system (1.7), and abstract results for delayed recursion. In Section 3, we prove the existence of traveling wave solutions using our abstract result in [7]. Some concluding remarks are given in Section 4.

\section{Some Preliminaries}

\subsection{Equilibria and stability}

Based on the model (1.7), the corresponding nonspatial system is yield:

$$
\begin{aligned}
& u_{, t}=\alpha_{1} u\left(t-\tau_{1}\right)-\eta_{1} u^{2}-p_{1} u v, \\
& v_{, t}=\alpha_{2} v\left(t-\tau_{2}\right)-\eta_{2} v^{2}-p_{2} u v
\end{aligned}
$$

with initial conditions

$$
u(t), v(t)>0 \quad \text { for }-\tau_{i} \leq t \leq 0 .
$$

It is straightforward to show that the solutions of system (2.1), subject to (2.2) above, satisfy $u(t), v(t)>0$ on $(0, \infty)$. Positivity of $(2.1)$ is important for both the modeling and the analysis. From the point of biology, positivity implies that the system persists. 
Table 2. Summary of existence and local stability criteria of equilibria.

\begin{tabular}{lll}
\hline Steady state & Criteria for existence & Criteria for stability \\
\hline$E_{0}$ & always exists & unstable \\
$E_{u}$ & always exists & $\alpha_{1} p_{2}>\alpha_{2} \eta_{1}$ \\
$E_{v}$ & always exists & $\alpha_{2} p_{1}>\alpha_{1} \eta_{2}$ \\
$E_{+}$ & $\alpha_{2} p_{1}<\alpha_{1} \eta_{2}, \alpha_{1} p_{2}<\alpha_{2} \eta_{1}$ & $\alpha_{1} p_{2}<\alpha_{2} \eta_{1}$ and $\alpha_{2} p_{1}<\alpha_{1} \eta_{2}$ \\
& or $\alpha_{2} p_{1}>\alpha_{1} \eta_{2}, \alpha_{1} p_{2}>\alpha_{2} \eta_{1}$ & \\
\hline
\end{tabular}

System (2.1) has the trivial equilibrium $E_{0}=(0,0)$, the mono-culture equilibria $E_{u}=\left(u^{*}, 0\right)$ and $E_{v}=\left(0, v^{*}\right)$ with

$$
u^{*}=\frac{\alpha_{1}}{\eta_{1}}, \quad v^{*}=\frac{\alpha_{2}}{\eta_{2}},
$$

and the coexistence equilibrium $E_{+}=\left(u_{+}, v_{+}\right)$with

$$
u_{+}=\frac{\alpha_{2} p_{1}-\alpha_{1} \eta_{2}}{p_{1} p_{2}-\eta_{1} \eta_{2}}, \quad v_{+}=\frac{\alpha_{1} p_{2}-\alpha_{2} \eta_{1}}{p_{1} p_{2}-\eta_{1} \eta_{2}}
$$

Clearly, $E_{+}$exists if and only if $\alpha_{2} p_{1}<\alpha_{1} \eta_{2}$ and $\alpha_{1} p_{2}<\alpha_{2} \eta_{1}$ or $\alpha_{2} p_{1}>\alpha_{1} \eta_{2}$ and $\alpha_{1} p_{2}>\alpha_{2} \eta_{1}$.

We summarize the local stability results for nonspatial system (2.1) in Table 2. The following global stability results can be found in Zhang, Li, Shang [22] or Al-Omari and Gourley [2].

Theorem 1. Let $E_{u}, E_{v}$ and $E_{+}$be defined as above, then the following statements are valid:

i. If $\alpha_{1} p_{2}>\alpha_{2} \eta_{1}, \alpha_{2} p_{1}<\alpha_{1} \eta_{2}$, then the mono-culture equilibrium $E_{u}$ is globally asymptotically stable;

ii. If $\alpha_{1} p_{2}<\alpha_{2} \eta_{1}, \alpha_{2} p_{1}>\alpha_{1} \eta_{2}$, then the mono-culture equilibrium $E_{v}$ is globally asymptotically stable;

iii. If $\alpha_{1} p_{2}<\alpha_{2} \eta_{1}, \alpha_{2} p_{1}<\alpha_{1} \eta_{2}$, then the unique coexistence equilibrium $E_{+}$ is globally asymptotically stable.

By the study of the local and global stability of the equilibria of the nonspatial system (2.1), we conclude that:

1. At least one of two interactive species with stage structure can persist in a stream due to the fact that the trivial equilibrium $E_{0}$ is always unstable.

2. One species out-competes the other one. In other words, one of them will die out due to the competition for the limited source in the long run.

3. However, under the conditions that the two mono-culture equilibria $E_{u}$ and $E_{v}$ are both unstable, the two species can coexist and approach a stable population density in the long term. This is the explanation of the fact that the unique coexistence equilibrium $E_{+}$is globally asymptotically stable under certain conditions given in the Theorem 1 part iii. 


\subsection{Abstract result for delayed recursion}

We use $\mathcal{H}$ to denote the habitat where the species grow, interact and migrate. $\mathcal{H}$ is either the real line (the continuous habitat) or the subset of the real line which consists of all integral multiples of positive mesh size $h$ (a discrete habitat). Let $\tau$ be a nonnegative real number. We shall use boldface Roman symbols like $\mathbf{u}(\theta, x)$ to denote $k$-vector-valued functions of the two variable $\theta$ and $x$, and boldface Greek letters to stand for $k$-vectors, which may be thought of as constant vector-valued functions. We define $\mathbf{u} \geq \mathbf{v}$ to mean that $u^{i}(\theta, x) \geq v^{i}(\theta, x)$ for all $i=1,2, \ldots, k, \theta \in[-\tau, 0]$ and $x \in \mathcal{H}$, and $\mathbf{u} \gg \mathbf{v}$ to mean that $u^{i}(\theta, x)>v^{i}(\theta, x)$ for all $i, \theta$ and $x$. We also define $\max \{\mathbf{u}(\theta, x), \mathbf{v}(\theta, x)\}$ to mean the vector-valued function whose $i$ th component at $(\theta, x)$ is $\max \left\{u^{i}(\theta, x), v^{i}(\theta, x)\right\}$. We use the notation $\mathbf{0}$ for the constant vector all of whose components are 0 .

Let $\mathcal{C}$ be the set of all bounded continuous functions from $[-\tau, 0] \times \mathcal{H}$ to $\mathbb{R}^{k}$, $\overline{\mathcal{C}}$ be the set of all bounded continuous functions from $[-\tau, 0]$ to $\mathbb{R}^{k}$, and $\mathcal{X}$ be the set of bounded continuous functions from $\mathcal{H}$ to $\mathbb{R}^{k}$. If $\mathbf{r} \in \overline{\mathcal{C}}$ with $\mathbf{r} \gg \mathbf{0}$, we define the set of continuous functions

$$
\mathcal{C}_{\mathbf{r}}:=\{\mathbf{u} \in \mathcal{C}: \mathbf{0} \leq \mathbf{u} \leq \mathbf{r}\}
$$

Moreover, we define the metric function $d(\cdot, \cdot)$ in $\mathcal{C}$ by

$$
d(\phi, \psi)=\sum_{k=0}^{\infty} \frac{\max _{|x| \leq k, \theta \in[-\tau, 0]}|\boldsymbol{\phi}(\theta, x)-\boldsymbol{\psi}(\theta, x)|}{2^{k}} \quad \forall \boldsymbol{\phi}, \boldsymbol{\psi} \in \mathcal{C}
$$

so that $(\mathcal{C}, d)$ is a metric space. The convergence of a sequence $\phi_{n}$ to $\phi$ with respect to this topology is equivalent to the uniform convergence of $\phi_{n}$ to $\phi$ on bounded subsets of $[-\tau, 0] \times \mathcal{H}$.

We study the following discrete-time recursion

$$
\mathbf{u}_{n+1}=Q\left[\mathbf{u}_{n}\right], \quad n=0,1,2, \ldots,
$$

where $\mathbf{u}_{n}(\theta, x)=\left(u_{n}^{1}(\theta, x), u_{n}^{2}(\theta, x), \ldots, u_{n}^{k}(\theta, x)\right), \theta \in[-\tau, 0]$, and $x \in \mathcal{H}$ represents the population densities of the populations of $k$ species at time $n$ and point $x$ with time delay $\tau$. The operator $Q$ is said to be order - preserving if $\mathbf{u} \geq \mathbf{v}$ implies that $Q[\mathbf{u}] \geq Q[\mathbf{v}]$. A recursion (2.4) in which $Q$ has this property is said to be cooperative. A function is said to be an equilibrium of $Q$ if $Q[\mathbf{w}]=\mathbf{w}$, so that if $\mathbf{u}_{l}=\mathbf{w}$ in the recursion (2.4), then $\mathbf{u}_{n}=\mathbf{w}$ for all $n \geq l$. We shall study the evolution of the solution $\mathbf{u}_{n}$ of the recursion (2.4) from a $\mathbf{u}_{0}$ near an unstable constant equilibrium $\boldsymbol{\theta}$. By introducing the new variable $\hat{\mathbf{u}}=\mathbf{u}-\boldsymbol{\theta}$ if necessary, we shall assume the unstable equilibrium $\boldsymbol{\theta}$ from which the system moves away is the origin $\mathbf{0}$.

We define the translation operators

$$
T_{y}[\mathbf{v}](\theta, x)=\mathbf{v}(\theta, x-y) .
$$

A set $D \in \mathcal{C}_{\mathbf{r}}$ is said to be $T$-invariant if $T_{y}[D]=D$ for any $y \in \mathcal{H}$.

We shall make the following hypotheses on $Q$. 


\section{Hypotheses 2.1}

i. $Q[\mathbf{0}]=\mathbf{0}$, and there is a vector $\boldsymbol{\beta}(\theta) \in \overline{\mathcal{C}}$ with $\boldsymbol{\beta}(\theta) \gg 0$ such that $Q[\boldsymbol{\beta}]=$ $\boldsymbol{\beta}$, and if $\mathbf{u}_{0}$ is any vector in $\overline{\mathcal{C}}$ with $\boldsymbol{\beta}(\theta) \gg \mathbf{u}_{0} \gg \mathbf{0}$, then the vector-valued function $\mathbf{u}_{n}$ obtained from the recursion (2.4) converges to $\boldsymbol{\beta}(\theta)$ uniformly on $[-\tau, 0]$ as $n$ approach infinity.

ii. $Q$ is order-preserving on nonnegative functions.

iii. $Q$ is translation invariant so that $Q\left[T_{y}[\mathbf{v}]\right]=T_{y}[Q[\mathbf{v}]]$ for all $y$.

iv. $Q$ is continuous with respect to the topology determined by $d(\cdot, \cdot)$ given in (2.3).

v. One of the following two properties holds:

a. $Q\left[\mathcal{C}_{\boldsymbol{\beta}}\right]$ is precompact in $\mathcal{C}_{\boldsymbol{\beta}}$.

b. The set $Q\left[\mathcal{C}_{\boldsymbol{\beta}}\right](0, \cdot)$ is precompact in $\mathcal{X}$, and there is a positive number $\zeta \leq \tau$ such that $Q[\mathbf{u}](\theta, x)=\mathbf{u}(\theta+\zeta, x)$ for all $\theta \in[-\tau,-\zeta]$, and the operator

$$
S[\mathbf{u}](\theta, x)= \begin{cases}\mathbf{u}(0, x), & \theta \in[-\tau,-\zeta), \\ Q[\mathbf{u}](\theta, x), & \theta \in[-\zeta, 0]\end{cases}
$$

has the property that $S[D]$ is precompact in $\mathcal{C}_{\boldsymbol{\beta}}$ for any $T$-invariant set $D \in \mathcal{C}_{\boldsymbol{\beta}}$ with $D(0, \cdot)$ precompact in $\mathcal{X}$.

Remark 1. Hypotheses $2.1 \mathrm{i}$-ii imply that $Q$ takes $\mathcal{C}_{\boldsymbol{\beta}}$ into itself, and that the equilibrium $\boldsymbol{\beta}$ attracts all initial functions in $\mathcal{C}_{\boldsymbol{\beta}}$ with uniformly positive components. In biological terms, $\boldsymbol{\beta}$ is a globally stable coexistence equilibrium. There may also be other equilibria lying between $\boldsymbol{\beta}$ and the extinction equilibrium $\mathbf{0}$, in each of which at least one of the species is extinct. Throughout this paper, we shall assume that the recursion (2.4) has a finite number of equilibria and that the equilibria of (2.4) are completely separate in the sense that for any two equilibria $\nu_{\mathbf{1}}(\theta), \nu_{\mathbf{2}}(\theta) \in \overline{\mathcal{C}}$ of $(2.4)$, if $\nu_{1}^{i}(\theta) \neq \nu_{2}^{i}(\theta)$ for some $\theta \in[-\tau, 0]$ then $\nu_{1}^{i}(\theta) \neq \nu_{2}^{i}(\theta)$ for all $\theta \in[-\tau, 0]$.

Remark 2. Here we have dropped the hypothesis in [7] that operator $Q$ is reflect invariant. In [7] the reflection invariance was assumed, but it was not used in the proof of Theorem 2.1 and Theorem 2.2. Consequently, Theorem 2.1 and Theorem 2.2 are still valid without the reflection assumption.

The following lemma can be found in Lui [9], Weinberger et. al [19], and Liang and Zhao [8].

Lemma 1 [Comparison Lemma]. Let $R$ be an order preserving operator. If $\mathbf{u}_{n}$ and $\mathbf{v}_{n}$ satisfy the inequalities $\mathbf{u}_{n} \leq R\left[\mathbf{u}_{n}\right]$ and $\mathbf{v}_{n} \geq R\left[\mathbf{v}_{n}\right]$ for all $n$, and if $\mathbf{u}_{0} \leq \mathbf{v}_{0}$, then $\mathbf{u}_{n} \leq \mathbf{v}_{n}$ for all $n$.

We choose a continuous vector-valued function $\phi(\theta, x)=\left(\phi^{1}, \ldots, \phi^{k}\right) \in \mathcal{C}_{\boldsymbol{\beta}}$ that has the properties

(b1) $\phi^{i}(\theta, x)$ is nonincreasing in $x$ for any $\theta \in[-\tau, 0]$ and $1 \leq i \leq k$; 
(b2) $\phi^{i}(\theta, x)=0$ for any $\theta \in[-\tau, 0], x \geq 0$, and $1 \leq i \leq k$;

(b3) $\phi(\theta,-\infty)=\alpha(\theta)$ for any $\theta \in[-\tau, 0]$, where $\mathbf{0} \ll \alpha(\theta) \ll \boldsymbol{\beta}$.

For any real number $c$ and $\mathbf{u} \in \mathcal{C}_{\boldsymbol{\beta}}$. Define the operator

$$
R_{c}[\mathbf{u}](\theta, s)=\max \left\{\phi(\theta, s), T_{-c}[Q[\mathbf{u}]](\theta, s)\right\} .
$$

Define a sequence of vector-valued functions $\mathbf{a}_{n}(c ; \theta, s)$ of $(\theta, s) \in[-\tau, 0] \times \mathcal{H}$ by the recursion

$$
\mathbf{a}_{n+1}(c ; \theta, s)=R_{c}\left[\mathbf{a}_{n}(c ; \cdot)\right](\theta, s), \quad \mathbf{a}_{0}(c ; \theta, s)=\phi(\theta, s) .
$$

Denote $\mathbf{a}(c ; \theta, \infty)$ as the limit of $\mathbf{a}_{n}(c ; \theta, \infty)$. Note that $\mathbf{a}_{n} \leq \mathbf{a}_{n+1} \leq \boldsymbol{\beta}$ for all $n$, and $\mathbf{a}_{n}(c ; \theta, s)$ is nonincreasing in $c$ and $s$ and continuous in $(c, \theta, s)$.

Define

$$
c^{*}:=\sup \{c: \mathbf{a}(c ; \theta, \infty) \neq \mathbf{0}\} .
$$

Denote $\left\{Q_{t}\right\}_{t=0}^{\infty}$ as the continuous time semiflow on $\mathcal{C}_{\boldsymbol{\beta}}$. In [7] we established the following existence result (see [7, Theorem 2.2]).

Theorem 2. Suppose that for any $t>0, Q_{t}$ satisfies Hypotheses 2.1. Let $c^{*}$ be given by (2.6) where $Q$ is replaced by $Q_{1}$. Then the following statements are true for $\left\{Q_{t}\right\}_{t=0}^{\infty}$.

i. If $c \geq c^{*}$, then there is a nonincreasing travelling wave solution $\mathbf{W}(c ; \theta, x-c t)$ of speed $c$ with $\mathbf{W}(c ; \theta, \infty)=\mathbf{0}$ and $\mathbf{W}(c ; \theta,-\infty)$ an equilibrium other than $\mathbf{0}$.

ii. If there is a nonincreasing travelling wave $\mathbf{W}(c ; \theta, x-c t)$ with $\mathbf{W}(c ; \theta, \infty)=\mathbf{0}$ and $\mathbf{W}(c ; \theta,-\infty)=\boldsymbol{\beta}$, then $c \geq c^{*}$.

We shall employ this abstract result to prove the existence of travelling wave solutions in model (1.7).

\section{Existence of Traveling Waves in (1.7)}

Suppose that

$$
\alpha_{1} p_{2}<\alpha_{2} \eta_{1}
$$

By Theorem $1, E_{u}$ is unstable, $E_{+}$exists and it is globally attracting if $\alpha_{2} p_{1}<$ $\alpha_{1} \eta_{2}$, and $E_{+}$does not exist and $E_{v}$ is globally attracting if $\alpha_{2} p_{1}>\alpha_{1} \eta_{2}$. We shall study the existence of travelling wave solutions connecting $E_{u}$ to $E_{+}$if $\alpha_{2} p_{1}<\alpha_{1} \eta_{2}$, and to $E_{v}$ if $\alpha_{2} p_{1}>\alpha_{1} \eta_{2}$, see Figure 1 .

Let $u:=u^{*}-u$ and $v:=v$. We convert the competition system (1.7) into the following cooperative system

$$
\begin{aligned}
& u_{, t}=d_{1} u_{, x x}-a_{1} u_{, x}-\alpha_{1}\left(u^{*}-u\left(t-\tau_{1}, x\right)\right)+\eta_{1}\left(u^{*}-u\right)^{2}+p_{1}\left(u^{*}-u\right) v \\
& v_{, t}=d_{2} v_{, x x}-a_{2} v_{, x}+\alpha_{2} v\left(t-\tau_{2}, x\right)-\eta_{2} v^{2}-p_{2}\left(u^{*}-u\right) v
\end{aligned}
$$




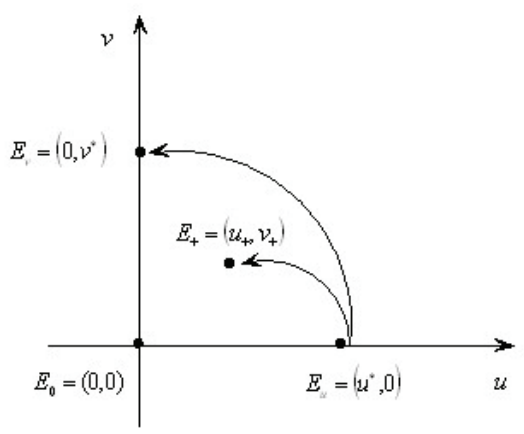

Figure 1. The spatial transition from $E_{u}$ to $E_{v}$ if $\alpha_{2} p_{1}>\alpha_{1} \eta_{2}$, and to $E_{+}$if $\alpha_{2} p_{1}<\alpha_{1} \eta_{2}$ in competition system (1.7).

For this system, we denote

$$
\boldsymbol{\beta}=(\tilde{u}, \tilde{v})= \begin{cases}\left(u^{*}-u^{+}, v^{+}\right), & \text {if } \alpha_{2} p_{1}<\alpha_{1} \eta_{2} \\ \left(u^{*}, v^{*}\right), & \text { if } \alpha_{2} p_{1}>\alpha_{1} \eta_{2}\end{cases}
$$

Clearly, $\mathbf{0}=(0,0)$ and $\boldsymbol{\beta}$ are the only two equilibria in $\mathcal{C}_{\boldsymbol{\beta}}$ if $\alpha_{2} p_{1}<\alpha_{1} \eta_{2}$; there is an extra equilibrium $\boldsymbol{\nu}=\left(u^{*}, 0\right)$ in $\mathcal{C}_{\boldsymbol{\beta}}$ if $\alpha_{2} p_{1}>\alpha_{1} \eta_{2}$. Hence, we now only to prove the existence of travelling wave solutions connecting $\mathbf{0}$ to $\boldsymbol{\beta}$, see Figure 2 .

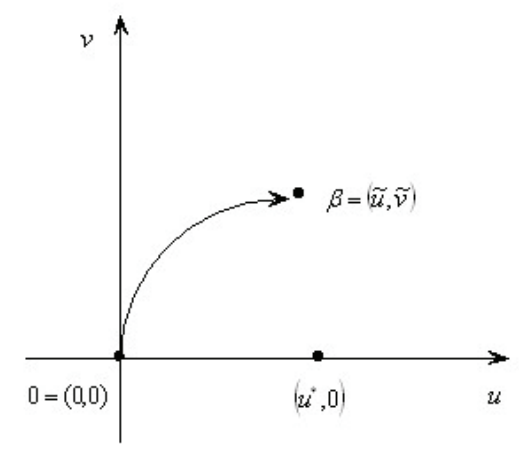

Figure 2. The spatial transition from $\mathbf{0}$ to $\boldsymbol{\beta}$ in cooperative system (3.2).

We now state and prove our main result.

Theorem 3. Assume that the condition (3.1) holds. Let $\boldsymbol{\beta}$ be defined by (3.3), and $c^{*}$ be defined by (2.6) where $Q$ is the time one solution map $Q_{1}$ of (3.2). Then system (3.2) has a nonincreasing traveling wave solution $\mathbf{W}(x-c t)=$ $(u(x-c t), v(x-c t))$ with $\mathbf{W}(+\infty)=\mathbf{0}$ and $\mathbf{W}(-\infty)=\boldsymbol{\beta}$ if and only if $c \geq c^{*}$. 
Remark 3. When the advection terms $a_{i}=0, i=1,2$, model (1.7) reduces to model (1.4). Theorem 3 indicates that the technical assumption made in [2] is indeed unnecessary and $c^{*}$ is the slowest travelling wave speed.

In what follows we shall prove Theorem 3 by using Theorem 2, that is verifying the abstract Hypotheses 2.1.

Let $\tau=\max \left\{\tau_{1}, \tau_{2}\right\}$. Define $f_{i}: \mathcal{C} \times \mathcal{C} \rightarrow \mathcal{X}, i=1,2$, by

$$
\begin{aligned}
f_{1}(\phi, \psi)(x)= & -\alpha_{1}\left(u^{*}-\phi\left(-\tau_{1}, x\right)\right)+\eta_{1}\left(u^{*}-\phi(0, x)\right)^{2} \\
& +p_{1}\left(u^{*}-\phi(0, x)\right) \psi(0, x), \\
f_{2}(\phi, \psi)(x)= & \alpha_{2} \psi\left(-\tau_{2}, x\right)-\eta_{2} \psi(0, x)^{2}-p_{2}\left(u^{*}-\phi(0, x)\right) \psi(0, x) .
\end{aligned}
$$

Then system (3.2) can be rewritten as

$$
\begin{aligned}
& u_{, t}=d_{1} u_{, x x}-a_{1} u_{, x}+f_{1}\left(u_{t}, v_{t}\right)(x), \\
& v_{, t}=d_{2} v_{, x x}-a_{2} v_{, x}+f_{2}\left(u_{t}, v_{t}\right)(x),
\end{aligned}
$$

where $t>0, x \in \mathbb{R}, u_{t}, v_{t} \in \mathcal{C}$ with $u_{t}(\theta, x)=u(t+\theta, x)$ and $v_{t}(\theta, x)=v(t+\theta, x)$ for $\theta \in[-\tau, 0], x \in \mathbb{R}$.

Definition 1. A function $(u(t, x), v(t, x)):[-\tau, b) \times \mathbb{R} \rightarrow \mathbb{R}^{2}, b>0$, with the properties that $(u, v)$ is $C^{2}$ in $x \in \mathbb{R}$ and $C^{1}$ in $t \in(0, b)$ is called a supersolution (subsolution) of $(3.2)$ on $[0, b)$ if for $t \in[0, b), x \in \mathbb{R}$

$$
\begin{aligned}
& u_{, t} \leq(\geq) d_{1} u_{, x x}-a_{1} u_{, x}+f_{1}\left(u_{t}, v_{t}\right)(x), \\
& v_{, t} \leq(\geq) d_{2} v_{, x x}-a_{2} v_{, x}+f_{2}\left(u_{t}, v_{t}\right)(x) .
\end{aligned}
$$

Lemma 2. If initial function $(\phi, \psi) \in \mathcal{C}_{\boldsymbol{\beta}}$, then system (3.2) has a unique classical solution $(u(t, x ; \phi, \psi), v(t, x ; \phi, \psi))$ on $(\tau, \infty) \times \mathbb{R}$, where $(u(0, x ; \phi, \psi)$, $v(0, x, ; \phi, \psi))=(\phi, \psi)$. If super-solution $(\bar{u}(t, x), \bar{v}(t, x))$ and sub-solution $(\underline{u}(t, x), \underline{v}(t, x))$ of $(3.2)$ satisfy $\mathbf{0} \leq(\underline{u}(t, x), \underline{v}(t, x)) \leq(\bar{u}(t, x), \bar{v}(t, x)) \leq \boldsymbol{\beta}$ for $t \in[-\tau, 0)$ and $x \in \mathbb{R}$, then $\mathbf{0} \leq(\underline{u}(t, x), \underline{v}(t, x)) \leq(\bar{u}(t, x), \bar{v}(t, x)) \leq \boldsymbol{\beta}$ for $t \geq 0$ and $x \in \mathbb{R}$.

Proof. Let $\left\{T_{u}(t)\right\}_{t \geq 0}$ and $\left\{T_{v}(t)\right\}_{t \geq 0}$ be the solution semigroup on $\mathcal{X}$ generated by the heat equations

$$
u_{, t}=d_{1} u_{, x x}-a_{1} u_{, x} \quad \text { and } \quad v_{, t}=d_{2} v_{, x x}-a_{2} v_{, x} .
$$

Then we can write (3.5) as the following integral equations

$$
\begin{aligned}
& u(t, x)=T_{u}(t) u(0, \cdot)(x)+\int_{0}^{t} T_{u}(t-s) f_{1}\left(u_{s}, v_{s}\right)(x) d s \\
& v(t, x)=T_{v}(t) v(0, \cdot)(x)+\int_{0}^{t} T_{v}(t-s) f_{2}\left(u_{s}, v_{s}\right)(x) d s
\end{aligned}
$$

where

$$
T_{u}(t)=\frac{1}{\sqrt{4 \pi d_{1} t}} \int_{\mathbb{R}} e^{-\frac{\left.\left(y-x+a_{1} t\right)\right)^{2}}{4 d_{1} t}} d y, \quad t>0
$$


and

$$
T_{v}(t)=\frac{1}{\sqrt{4 \pi d_{2} t}} \int_{\mathbb{R}} e^{-\frac{\left.\left(y-x+a_{2} t\right)\right)^{2}}{4 d_{2} t}} d y, \quad t>0 .
$$

Under the abstract setting in [11], a mild solution of (3.5) is a solution to its associated integral equation (3.6). One can easily verify that $f_{1}$ and $f_{2}$ are Lipschitz continuous on any bounded subset of $\mathcal{C} \times \mathcal{C}$. Let $\mathcal{Z}=B U C\left(\mathbb{R}, \mathbb{R}^{2}\right)$ be the Banach space of all bounded and uniformly continuous functions from $\mathbb{R}$ into $\mathbb{R}^{2}$ with the usual supremum norm. Let $\mathcal{Z}^{+}=\left\{\left(\phi_{1}, \phi_{2}\right):\left(\phi_{1}, \phi_{2}\right) \in \mathcal{Z}\right.$, $\left.\phi(x)_{i} \geq 0, i=1,2\right\}$. We show that $f_{1}$ and $f_{2}$ are quasi-monotone on $\mathcal{C}$ in the sense that

$$
\begin{aligned}
& \lim _{h \rightarrow 0^{+}} \frac{1}{h} \operatorname{dist}\left(\phi_{2}(0)-\phi_{1}(0)+h\left[f_{1}\left(\phi_{2}, \psi_{2}\right)-f_{1}\left(\phi_{1}, \psi_{1}\right)\right] ; \mathcal{Z}^{+}\right)=0, \\
& \lim _{h \rightarrow 0^{+}} \frac{1}{h} \operatorname{dist}\left(\psi_{2}(0)-\psi_{1}(0)+h\left[f_{2}\left(\phi_{2}, \psi_{2}\right)-f_{2}\left(\phi_{1}, \psi_{1}\right)\right] ; \mathcal{Z}^{+}\right)=0
\end{aligned}
$$

for all $\phi_{j}, \psi_{j} \in \mathcal{C}_{\boldsymbol{\beta}}, j=1,2$ with $\left(\phi_{2}, \psi_{2}\right) \geq\left(\phi_{1}, \psi_{1}\right)$. From the definitions of $f_{1}$ and $f_{2}$ in (3.4) we get

$$
\begin{aligned}
f_{1}\left(\phi_{2}, \psi_{2}\right)-f_{1}\left(\phi_{1}, \psi_{1}\right) \\
=-\alpha_{1}\left(u^{*}-\phi_{2}\left(-\tau_{1}, x\right)\right)+\eta_{1}\left(u^{*}-\phi_{2}(0, x)\right)^{2}+p_{1}\left(u^{*}-\phi_{2}(0, x)\right) \psi_{2}(0, x) \\
\quad+\alpha_{1}\left(u^{*}-\phi_{1}\left(-\tau_{1}, x\right)\right)-\eta_{1}\left(u^{*}-\phi_{1}(0, x)\right)^{2}-p_{1}\left(u^{*}-\phi_{1}(0, x)\right) \psi_{1}(0, x) \\
=\alpha_{1}\left(\phi_{2}\left(-\tau_{1}, x\right)-\phi_{1}(-\tau, x)\right)+p_{1}\left(\psi_{2}(0, x)-\psi_{1}(0, x)\right)\left(u^{*}-\phi_{1}(0, x)\right) \\
\quad+\left(\phi_{1}(0, x)-\phi_{2}(0, x)\right)\left[p_{1} \psi_{2}(0, x)+\eta_{1}\left(2 u^{*}-\phi_{1}(0, x)-\phi_{2}(0, x)\right)\right]
\end{aligned}
$$

and

$$
\begin{aligned}
f_{2}\left(\phi_{2}, \psi_{2}\right)-f_{2}\left(\phi_{1}, \psi_{1}\right) \\
=\alpha_{2} \psi_{2}\left(-\tau_{2}, x\right)-\eta_{2} \psi_{2}(0, x)^{2}-p_{2}\left(u^{*}-\phi_{2}(0, x)\right) \psi_{2}(0, x)-\alpha_{2} \psi_{1}\left(-\tau_{2}, x\right) \\
\quad+\eta_{2} \psi_{1}(0, x)^{2}+p_{2}\left(u^{*}-\phi_{1}(0, x)\right) \psi_{1}(0, x) \\
=\alpha_{2}\left(\psi_{2}\left(-\tau_{2}, x\right)-\psi_{1}\left(-\tau_{2}, x\right)\right)+p_{2}\left(\phi_{2}(0, x) \psi_{2}(0, x)-\phi_{1}(0, x) \psi_{1}(0, x)\right) \\
\quad+\left(\psi_{1}(0, x)-\psi_{2}(0, x)\right)\left[\eta_{2}\left(\psi_{1}(0, x)+\psi_{2}(0, x)\right)+p_{2} u^{*}\right] .
\end{aligned}
$$

It follows that for sufficiently small $h>0$,

$$
\begin{aligned}
& \phi_{2}(0, x)-\phi_{1}(0, x)+h\left[f_{1}\left(\phi_{2}, \psi_{2}\right)-f_{1}\left(\phi_{1}, \psi_{1}\right)\right] \\
& \quad=h \alpha_{1}\left(\phi_{2}\left(-\tau_{1}, x\right)-\phi_{1}(-\tau, x)\right)+h p_{1}\left(\psi_{2}(0, x)-\psi_{1}(0, x)\right)\left(u^{*}-\phi_{1}(0, x)\right) \\
& \quad+\left(\phi_{2}(0, x)-\phi_{1}(0, x)\right)\left[1-h\left(\eta_{1}\left(2 u^{*}-\phi_{1}(0, x)-\phi_{2}(0, x)\right)+p_{1} \psi_{2}(0, x)\right)\right] \\
& \quad \geq 0
\end{aligned}
$$

and

$$
\begin{aligned}
& \psi_{2}(0, x)-\psi_{1}(0, x)+h\left[f_{2}\left(\phi_{2}, \psi_{2}\right)-f_{2}\left(\phi_{1}, \psi_{1}\right)\right] \\
& =h \alpha_{2}\left(\psi_{2}\left(-\tau_{2}, x\right)-\psi_{1}\left(-\tau_{2}, x\right)\right)+h p_{2}\left(\phi_{2}(0, x) \psi_{2}(0, x)-\phi_{1}(0, x) \psi_{1}(0, x)\right) \\
& \quad+\left(\psi_{2}(0, x)-\psi_{1}(0, x)\right)\left[1-h\left(\eta_{2}\left(\psi_{1}(0, x)+\psi_{2}(0, x)\right)+p_{2} u^{*}\right)\right] \\
& \quad \geq 0 .
\end{aligned}
$$


Which indicate that (3.7) holds. By Corollary 5 in [11], we can show the existence and uniqueness of $(u(t, x ; \phi, \psi), v(t, x ; \phi, \psi))$ with

$$
\begin{aligned}
& \left(S_{1}(t, s), S_{2}(t, s)\right)=\left(S_{1}(t-s), S_{2}(t-s)\right), \\
& \left(T_{1}(t, s), T_{2}(t, s)\right)=\left(T_{1}(t-s), T_{2}(t-s)\right)
\end{aligned}
$$

for $t \geq s \geq 0$,

$$
\left(B_{1}(t, \phi, \psi), B_{2}(t, \phi, \psi)\right)=\left(f_{1}(\phi, \psi), f_{2}(\phi, \psi)\right),
$$

and $v^{+}=\boldsymbol{\beta}, v^{-}=\mathbf{0}$. Moreover, by the semigroup theory given in the proof of Theorem 1 in [11], it follows that $(u(t, x ; \phi), v(t, x ; \psi))$ is a classical solution for $t>\tau$.

Let $\boldsymbol{\Psi}(\theta, x)=(\bar{u}(\theta, x), \bar{v}(\theta, x)), \boldsymbol{\Phi}(\theta, x)=(\underline{\mathrm{u}}(\theta, x), \underline{\mathrm{v}}(\theta, x))$, for $\theta \in[-\tau, 0]$, $x \in \mathbb{R}$. Then $\mathbf{0} \leq \boldsymbol{\Phi} \leq \boldsymbol{\Psi} \leq \boldsymbol{\beta}$ with $\boldsymbol{\Phi} \leq \boldsymbol{\Psi}$ in $\mathcal{C}_{\boldsymbol{\beta}}$. Again by Corollary 5 in [11], we have

$$
\mathbf{0} \leq(u(t, x, \boldsymbol{\Phi}), v(t, x, \boldsymbol{\Phi})) \leq(u(t, x, \boldsymbol{\Psi}), v(t, x, \boldsymbol{\Psi})) \leq \boldsymbol{\beta} .
$$

for $t \geq 0, x \in \mathbb{R}$.

Let $v^{+}=\boldsymbol{\beta}$ and $v^{-}=(\underline{\mathrm{u}}(t, x), \underline{\mathrm{v}}(t, x)), v^{+}=(\bar{u}(t, x), \bar{v}(t, x))$ and $v^{-}=\mathbf{0}$, respectively, we obtain

$$
\begin{aligned}
& (\underline{\mathrm{u}}(t, x), \underline{\mathrm{v}}(t, x)) \leq(u(t, x, \boldsymbol{\Phi}), v(t, x, \boldsymbol{\Phi})) \leq \boldsymbol{\beta}, \quad t \geq 0, x \in \mathbb{R}, \\
& \mathbf{0} \leq(u(t, x, \boldsymbol{\Psi}), v(t, x, \boldsymbol{\Psi})) \leq(\bar{u}(t, x), \bar{v}(t, x)), \quad t \geq 0, x \in \mathbb{R} .
\end{aligned}
$$

It follows from $(3.8)-(3.10)$ that $(\bar{u}(t, x), \bar{v}(t, x)) \geq(\underline{\mathrm{u}}(t, x), \underline{\mathrm{v}}(t, x))$ for all $t \geq 0$, $x \in \mathbb{R}$. This completes the proof.

Lemma 2 together with the Theorem 1 shows that the time $t$ solution map $Q_{t}$ of system (3.2) with $t>0$ exists, and it satisfies Hypotheses $2.1 i-i i$. Hypotheses 2.1 iii is satisfied by $Q_{t}$ since system (3.2) is autonomous.

Lemma 3. For any $t>0, Q_{t}$ satisfies Hypothesis 2.1 iv with $\boldsymbol{\beta}$ given by (3.3).

Proof. Let $\Phi:=(\phi, \psi), \Phi_{1}:=\left(\phi_{1}, \psi_{1}\right), \Phi_{2}:=\left(\phi_{2}, \psi_{2}\right) \in \mathcal{C}_{\boldsymbol{\beta}}$. For any $\varepsilon>0$ and $t_{0}>0$, we define

$$
\begin{aligned}
& H(t, x):=\left|u\left(t, x ; \Phi_{1}\right)-u\left(t, x ; \Phi_{2}\right)\right|+\left|v\left(t, x ; \Phi_{1}\right)-v\left(t, x ; \Phi_{2}\right)\right| ; \\
& K:=\sup _{t \in\left[0, t_{0}\right], x \in \mathbb{R}} H(t, x) ; \quad \Omega_{r}(z):=[-\tau, 0] \times[z-r, z+r], \quad \forall r>0, z \in \mathbb{R} ; \\
& |\phi|_{\Omega_{r}(z)}:=\sup _{(\theta, x) \in \Omega_{r}(z)}|\phi(\theta, x)| ; \quad \epsilon_{0}:=\frac{\varepsilon}{2(3+\Delta) t_{0} e^{\Delta t_{0}}} ;
\end{aligned}
$$

where $\Delta:=\alpha_{1}+\alpha_{2}+4 \eta_{1} u^{*}+2 \eta_{2} v^{*}+\left(p_{1}+p_{2}\right)\left(2 u^{*}+v^{*}\right)$. Without loss of generality, we assume $K \geq \sup _{\theta \in[-\tau, 0], x \in \mathbb{R}} H(\theta, x)$. Then, there exists $\left(t^{*}, x^{*}\right) \in$ $\left[0, t_{0}\right] \times \mathbb{R}$ such that $H(\theta, x) \leq H\left(t^{*}, x^{*}\right)+\varepsilon_{0}$ for $(t, \theta, x) \in\left[0, t_{0}\right] \times[-\tau, 0] \times \mathbb{R}$. We choose $\sigma=\varepsilon / 4 e^{\Delta t_{0}}$ and $M=M\left(\varepsilon, t_{0}\right)>0$ such that for any $t \in\left[0, t_{0}\right]$

$$
\begin{aligned}
& \int_{|y|>M} \frac{1}{\sqrt{4 \pi d_{1} t^{*}}} e^{-\frac{\left(y-x+a_{1} t\right)^{2}}{4 d_{1} t^{*}}} d y \leq \frac{\varepsilon_{0}}{u^{*}}, \quad \int_{|y|>M} \frac{1}{\sqrt{4 \pi d_{2} t^{*}}} e^{-\frac{\left(y-x+a_{2} t\right)^{2}}{4 d_{2} t^{*}}} d y \leq \frac{\varepsilon_{0}}{v^{*}}, \\
& T_{u}(t) H_{s}\left(0, x^{*}\right) \leq\left|H_{s}\right|_{\Omega_{M}\left(x^{*}\right)}+\varepsilon_{0}, \quad T_{v}(t) H_{s}\left(0, x^{*}\right) \leq\left|H_{s}\right|_{\Omega_{M}\left(x^{*}\right)}+\varepsilon_{0},
\end{aligned}
$$




$$
T_{u}(t) H_{s}\left(-\tau_{1}, x^{*}\right) \leq\left|H_{s}\right|_{\Omega_{M}\left(x^{*}\right)}+\varepsilon_{0}, \quad T_{v}(t) H_{s}\left(-\tau_{2}, x^{*}\right) \leq\left|H_{s}\right|_{\Omega_{M}\left(x^{*}\right)}+\varepsilon_{0} .
$$

For $i=1,2$ if $\left|\Phi_{1}(\theta, x)-\Psi_{1}(\theta, x)\right|_{\Omega_{M}\left(x^{*}\right)}<\sigma$, then

$$
\begin{aligned}
\left|u\left(t^{*}, x^{*} ; \Phi_{1}\right)-u\left(t^{*}, x^{*} ; \Phi_{2}\right)\right| & \mid T_{u}\left(t^{*}\right) u\left(0, x^{*} ; \Phi_{1}\right)+\int_{0}^{t^{*}} T_{u}\left(t^{*}-s\right) f_{1}\left(\left(u_{s}\left(\cdot, \cdot ; \Phi_{1}\right), v_{s}\left(\cdot, \cdot ; \Phi_{1}\right)\right)\left(x^{*}\right) d s\right. \\
& -T_{u}\left(t^{*}\right) u\left(0, x^{*} ; \Phi_{2}\right)-\int_{0}^{t^{*}} T_{u}\left(t^{*}-s\right) f_{1}\left(u_{s}\left(\cdot, \cdot ; \Phi_{2}\right), v_{s}\left(\cdot, \cdot ; \Phi_{2}\right)\right)\left(x^{*}\right) d s \mid \\
\leq & T_{u}\left(t^{*}\right)\left|u\left(0, x^{*} ; \Phi_{1}\right)-u\left(0, x^{*} ; \Phi_{2}\right)\right| \\
& +\int_{0}^{t^{*}} T_{u}\left(t^{*}-s\right) \mid f_{1}\left(\left(u_{s}\left(\cdot, \cdot ; \Phi_{1}\right), v_{s}\left(\cdot, \cdot ; \Phi_{1}\right)\right)\right. \\
& -f_{1}\left(u_{s}\left(\cdot, \cdot ; \Phi_{2}\right), v_{s}\left(\cdot, \cdot ; \Phi_{2}\right)\right) \mid\left(x^{*}\right) d s \\
\leq & T_{u}\left(t^{*}\right) H\left(0, x^{*}\right)+\alpha_{1} \int_{0}^{t^{*}} T_{u}\left(t^{*}-s\right) H_{s}\left(-\tau_{1}, x\right) d s \\
& +4 \eta_{1} u^{*} \int_{0}^{t^{*}} T_{u}\left(t^{*}-s\right) H_{s}\left(0, x^{*}\right) d s \\
& +p_{1}\left(2 u^{*}+v^{*}\right) \int_{0}^{t^{*}} T_{u}\left(t^{*}-s\right) H_{s}\left(0, x^{*}\right) d s \\
\leq & \sigma+\varepsilon_{0}+\alpha_{1} \int_{0}^{t_{0}}\left(\left|H_{s}\right|_{\Omega_{M}\left(x^{*}\right)}+\varepsilon_{0}\right) d s+4 \eta_{1} u^{*} \int_{0}^{t_{0}}\left(\left|H_{s}\right|_{\Omega_{M}\left(x^{*}\right)}+\varepsilon_{0}\right) d s \\
& +p_{1}\left(2 u^{*}+v^{*}\right) \int_{0}^{t_{0}}\left(\left|H_{s}\right|_{\Omega_{M}\left(x^{*}\right)}+\varepsilon_{0}\right) d s \\
= & +\varepsilon_{0}\left(1+\alpha_{1} t_{0}+4 \eta_{1} u^{*} t_{0}+p_{1}\left(2 u^{*}+v^{*}\right) t_{0}\right) \\
& +\left(\alpha_{1}+4 \eta_{1} u^{*}+p_{1}\left(2 u^{*}+v^{*}\right)\right) \int_{0}^{t_{0}}\left(\left|H_{s}\right|_{\Omega_{M}\left(x^{*}\right)}+\varepsilon_{0}\right) d s . \\
& \\
& \\
&
\end{aligned}
$$

By a similar argument, we have

$$
\begin{aligned}
& \left|v\left(t^{*}, x^{*} ; \Phi_{1}\right)-v\left(t^{*}, x^{*} ; \Phi_{2}\right)\right| \\
& \quad \leq \quad \sigma+\varepsilon_{0}\left(1+\alpha_{2} t_{0}+2 \eta_{2} u^{*} t_{0}+p_{2}\left(2 u^{*}+v^{*}\right) t_{0}\right) \\
& \quad+\left(\alpha_{2}+2 \eta_{1} v^{*}+p_{2}\left(2 u^{*}+v^{*}\right)\right) \int_{0}^{t_{0}}\left(\left|H_{s}\right|_{\Omega_{M}\left(x^{*}\right)}+\varepsilon_{0}\right) d s .
\end{aligned}
$$

We thus get

$$
\begin{aligned}
\left|H_{t}\right|_{\Omega_{M}\left(x^{*}\right)} & \leq \varepsilon_{0}+H\left(t^{*}, x^{*}\right) \\
& =\varepsilon_{0}+\left|u\left(t^{*}, x^{*} ; \Phi_{1}\right)-u\left(t^{*}, x^{*} ; \Phi_{2}\right)\right|+\left|v\left(t^{*}, x^{*} ; \Phi_{2}\right)-v\left(t^{*}, x^{*} ; \Phi_{2}\right)\right| \\
& \leq 2 \sigma+\varepsilon_{0}(3+\Delta) t_{0}+\Delta \int_{0}^{t_{0}}\left|H_{s}\right|_{\Omega_{M}\left(x^{*}\right)} d s .
\end{aligned}
$$

By Gronwall's inequality we get

$$
\left|H_{t}\right|_{\Omega_{M}^{u}\left(x^{*}\right)} \leq\left(2 \sigma+\varepsilon_{0}(3+\Delta) t_{0}\right) e^{\Delta t_{0}}, \quad \forall t \in\left[0, t_{0}\right] .
$$


We thus have obtained that for any $\varepsilon>0$, and compact subset $\mathcal{S} \subset[-\tau, 0] \times \mathbb{R}$, there exist $\sigma>0$ and a compact set $\Omega_{M}\left(x^{*}\right)$ such that $\mathcal{S} \subset \Omega_{M}\left(x^{*}\right)$ and

$$
\left|H_{t}\right|_{\mathcal{S}} \leq\left|H_{t}\right|_{\Omega_{M}\left(x^{*}\right)}<\varepsilon \quad \text { for } t \in\left[0, t_{0}\right] \quad \text { and } \quad\left|\Phi_{1}-\Phi_{2}\right|_{\Omega_{M}\left(x^{*}\right)}<\sigma .
$$

This shows that $Q_{t}$ is continuous in $\Phi$ with respect to the compact open topology uniformly for $t \in\left[0, t_{0}\right]$. Note that the metric space $\left(\mathcal{C}_{\boldsymbol{\beta}}, d\right)$ is complete. By the triangle inequality and the continuity of $Q_{t}$ in $t$ from Lemma 2, it then follows that $Q_{t}(\phi)$ is continuous in $(t ; \Phi)$ with respect to the compact open topology. Since (3.6) is an autonomous system, $\left\{Q_{t}\right\}_{t \geq 0}$ is a semiflow on $\mathcal{C}_{\boldsymbol{\beta}}$. This completes the proof of this Lemma.

Lemma 4. For any $t>0, Q_{t}$ satisfies Hypothesis $2.1 v$ with $\boldsymbol{\beta}$ given by (3.3).

Proof. Let $T(t)=\left(T_{u}(t), T_{v}(t)\right)$, where $T_{u}(t)$ is the solution map of $\frac{\partial u}{\partial t}=$ $d_{u} \frac{\partial^{2} u}{\partial x^{2}}-a_{1} \frac{\partial u}{\partial x}$ and $T_{v}(t)$ is the solution map of $\frac{\partial v}{\partial t}=d_{v} \frac{\partial^{2} v}{\partial x^{2}}-a_{2} \frac{\partial v}{\partial x}$. It follows that $\left\{T_{u}(t)\right\}_{t \geq 0}$ and $\left\{T_{v}(t)\right\}_{t \geq 0}$ are linear semigroup on $\mathcal{X}$ and $T_{u}(t)$ and $T_{v}(t)$ are compact for each $t>0$. Let $Q_{t}=\left(Q_{t}^{u}, Q_{t}^{v}\right)$. Given $t_{0}>\tau$, then

$$
\begin{aligned}
Q_{t_{0}}^{u}[(\phi, \psi)](\theta, x) & =u\left(t_{0}+\theta ; \phi, \psi\right) \\
& =T_{u}\left(t_{0}+\theta\right) \phi(0, \cdot)(x)+\int_{0}^{t_{0}+\theta} T_{u}\left(t_{0}+\theta-s\right) f_{1}\left(u_{s}, v_{s}\right)(x) d s
\end{aligned}
$$

and

$$
\begin{aligned}
Q_{t_{0}}^{v}[(\phi, \psi)](\theta, x) & =v\left(t_{0}+\theta ; \phi, \psi\right) \\
& =T_{v}\left(t_{0}+\theta\right) \phi(0, \cdot)(x)+\int_{0}^{t_{0}+\theta} T_{v}\left(t_{0}+\theta-s\right) f_{2}\left(u_{s}, v_{s}\right)(x) d s .
\end{aligned}
$$

By the properties of $T_{u}(t)$ and $T_{v}(t)$ and the boundness of $f_{i}$, we see that $Q_{t}^{u}$ and $Q_{t}^{v}$ are compact for each $t>\tau$. Thus $Q_{t}$ satisfies Hypotheses 2.1 v.a. when $t>\tau$.

Given $t_{0} \in(0, \tau]$, we now show that $Q_{t_{0}}$ satisfies Hypotheses 2.1 v.b. with $Q=Q_{t_{0}}$. To prove $S[D]$ is precompact, it suffices to show that for any given compact interval $I \subset \mathbb{R}, u(t, x ; \phi, \psi)$ and $v(t, x ; \phi, \psi)$ are equicontinuous in $(t, x) \in\left[0, t_{0}\right] \times I$ for all $(\phi, \psi) \in D$. We shall only show that $u(t, x ; \phi, \psi)$ is equicontinuous in $(t, x) \in\left[0, t_{0}\right] \times I$ for all $(\phi, \psi) \in D$, since the other statement can be proved similarly. By the continuity of integral, we have that for any $\varepsilon>0$, there exists $\delta_{0}>0$ such that for any $t \in\left(0, \delta_{0}\right]$,

$$
\left|\int_{0}^{t} T_{u}(t-s) f_{1}\left(u_{s}, v_{s}\right)(x) d s\right|<\frac{\varepsilon}{12} .
$$

On the other hand, since $D(0, \cdot)$ is precompact in $\mathcal{X}$, for the above interval $I$, there exists $\delta_{1}>0$ such that $\left|\phi\left(0, x_{1}\right)-\phi\left(0, x_{2}\right)\right|<\varepsilon / 6$ and $\mid \psi\left(0, x_{1}\right)-$ $\psi\left(0, x_{2}\right) \mid<\varepsilon / 6$ for all $(\phi, \psi) \in D$ when $x_{1}, x_{2} \in I$ with $\left|x_{1}-x_{2}\right|<\delta_{1}$. Thus for any $t_{1}, t_{2} \in\left[0, \delta_{0}\right], x_{1}, x_{2} \in I$ with $\left|x_{1}-x_{2}\right|<\delta_{1}$, we have

$$
\begin{gathered}
\left|u\left(t_{1}, x_{1} ; \phi, \psi\right)-u\left(t_{2}, x_{2} ; \phi, \psi\right)\right| \leq\left|u\left(t_{1}, x_{1} ; \phi, \psi\right)-\phi\left(0, x_{1}\right)\right| \\
+\left|u\left(t_{2}, x_{2} ; \phi, \psi\right)-\phi\left(0, x_{2}\right)\right|+\left|\phi\left(0, x_{1}\right)-\phi\left(0, x_{2}\right)\right| .
\end{gathered}
$$


We claim that $\left|u\left(t_{1}, x_{1} ; \phi, \psi\right)-\phi\left(0, x_{1}\right)\right|<\varepsilon / 6$ under appropriate choice of $\delta_{0}$. In fact, if we choose $M>0$ and $\delta_{0}$ properly so that

$$
\frac{\widetilde{u}}{\sqrt{\pi}} \int_{|y|>M} e^{-y^{2}} d y<\frac{\varepsilon}{48} \quad \text { and } \quad\left(\sqrt{4 d_{1} \delta_{0}}+a_{1} t_{1}\right) M<\delta_{1} .
$$

We then obtain that

$$
\begin{aligned}
\mid u( & \left.t_{1}, x_{1} ; \phi, \psi\right)-\phi\left(0, x_{1}\right) \mid \\
= & \left|T_{u}\left(t_{1}\right) \phi(0, \cdot)\left(x_{1}\right)+\int_{0}^{t_{1}} T_{u}\left(t_{1}-s\right) f_{1}\left(u_{s}, v_{s}\right)\left(x_{1}\right) d s-\phi\left(0, x_{1}\right)\right| \\
= & \mid \int_{\mathbb{R}} \frac{e^{-y^{2}}}{\sqrt{\pi}} \phi\left(0, x_{1}-a_{1} t_{1}-\sqrt{4 d_{u} t_{1}} y\right) d y \\
& +\int_{0}^{t_{1}} T_{u}\left(t_{1}-s\right) f_{1}\left(u_{s}, v_{s}\right)\left(x_{1}\right) d s-\phi\left(0, x_{1}\right) \mid \\
\leq & \int_{|y|>M \cup|y| \leq M} \frac{e^{-y^{2}}}{\sqrt{\pi}}\left|\phi\left(0, x_{1}-a_{1} t_{1}-\sqrt{4 d_{1} t_{1}} y\right)-\phi\left(0, x_{1}\right)\right| d y \\
& +\left|\int_{0}^{t_{1}} T_{u}\left(t_{1}-s\right) f_{1}\left(u_{s}, v_{s}\right) d s\right|<\frac{\varepsilon}{24}+\frac{\varepsilon}{24}+\frac{\varepsilon}{12}=\frac{\varepsilon}{6} .
\end{aligned}
$$

Similarly, we can show that $\left\|u\left(t_{2}, \phi, \psi\right)\right\|<\varepsilon / 6$ under appropriate choice of $\delta_{0}$. Hence we obtain that $\left|u\left(t_{1}, x_{1} ; \phi, \psi\right)-u\left(t_{2}, x_{2} ; \phi, \psi\right)\right|<\varepsilon / 2$. Since $Q_{t}^{u}$ is compact when $t>\tau$, it follows that $u(t, x ; \phi, \psi)$ is equicontinuous in $(t, x) \in\left[\delta_{0}, t_{0}\right] \times I$ for all $(\phi, \psi) \in D$. That is, for the above $\varepsilon$ and $I$, there exists $\delta_{2}>0$ so that $\left|u\left(t_{1}, x_{1} ; \phi, \psi\right)-u\left(t_{2}, x_{2} ; \phi, \psi\right)\right|<\varepsilon / 2$ for all $(\phi, \psi) \in D$ if $t_{1}, t_{2} \in\left[\delta_{0}, t_{0}\right]$ and $x_{1}, x_{2} \in I$ with $\left|t_{1}-t_{2}\right|+\left|x_{1}-x_{2}\right|<\delta_{2}$. Let $\delta:=$ $\min \left\{\delta_{0}, \delta_{1}, \delta_{2}\right\}$, then we have for any $\varepsilon>0$ and $I \in \mathbb{R}$, there exists $\delta>0$ such that $\left|u\left(t_{1}, x_{1} ; \phi, \psi\right)-u\left(t_{2}, x_{2} ; \phi, \psi\right)\right|<\varepsilon$ for all $(\phi, \psi) \in D$ if $t_{1}, t_{2} \in\left[0, t_{0}\right]$ and $x_{1}, x_{2} \in I$ with $\left|t_{1}-t_{2}\right|+\left|x_{1}-x_{2}\right|<\delta$. This completes the proof of this Lemma.

Lemma 5. Let $c^{*}$ be defined by (2.6) where $Q$ is replaced by the time one map $Q_{1}$ of $(3.2)$, then $0<c^{*}<\infty$.

Proof. We write the time one solution operator $Q_{1}$ of $(3.2)$ as $Q_{1}=\left(Q_{u 1}, Q_{v 1}\right)$. Let $\hat{Q}_{v 1}$ be the time one solution map of

$$
v_{, t}=d_{2} v_{, x x}-a_{2} v_{, x}+\alpha_{2} v\left(t-\tau_{2}, x\right)-\eta_{2} v^{2}-p_{2} u^{*} v
$$

The reaction term in $(3.11)$ is $f_{2}\left(0, v_{t}\right)(x)$ where $f_{2}$ given in (3.4). Since $f_{2}\left(u_{t}, v_{t}\right)(x) \geq f_{2}\left(0, v_{t}\right)(x), Q_{v 1}(u, v) \geq \hat{Q}_{v 1}(v)$ for $(u, v) \in \mathcal{C}_{\boldsymbol{\beta}}$. The definition of $c^{*}$ and Lemma 1 show that

$$
c^{*} \geq c_{v}^{*},
$$

where $c_{v}^{*}$ is given by (2.6) where $Q$ is $\hat{Q}_{v 1}$. 
The equation (3.11) has two constant equilibria $v=0$ and $v=\left(\alpha_{2} \eta_{1}-\right.$ $\left.\alpha_{1} p_{2}\right) /\left(\eta_{1} \eta_{2}\right)>0$ due to (3.1). One can verify that under the condition (3.1), 0 is unstable and $\left(\alpha_{2} \eta_{1}-\alpha_{1} p_{2}\right) /\left(\eta_{1} \eta_{2}\right)$ is stable. Since

$$
v_{, t} \leq d_{2} v_{, x x}-a_{2} v_{, x}+\alpha_{2} v\left(t-\tau_{2}, x\right)-p_{2} u^{*} v,
$$

the time $t$ solution map of (3.11) is dominated by that of the linearized model. One can then use Theorem 3.10 in Liang and Zhao [8] and the argument given on the pages 2758-2759 of Fang, Wei and Zhao [3] to find that

$$
c_{v}^{*}=\inf _{\mu>0} \lambda(\mu) / \mu
$$

where $\lambda(\mu)$ is the principal eigenvalue of the characteristic equation

$$
\eta_{1} \lambda-\eta_{1} d_{2} \mu^{2}-\eta_{1} a_{2} \mu-\eta_{1} \alpha_{2} e^{-\tau_{2} \lambda}+\alpha_{1} p_{2}=0 .
$$

By the equation (3.13) and the condition (3.1) one can easily show that $\lambda(\mu)>0$ for $\mu \geq 0$. On the other hand, $\lambda(\mu) \geq \eta_{1} d_{v} \mu^{2}$ for large positive $\mu$ so that $\lambda(\mu) / \mu \rightarrow \infty$ as $\mu \rightarrow \infty$. It follows that the infimum of $\lambda(\mu) / \mu$ occurs at a finite number and thus $c_{v}^{*}>0$. It follows from (3.12) that $c^{*}>0$.

We next show that $c^{*}<\infty$. From (3.2) we obtain

$$
\begin{aligned}
& u_{, t} \leq d_{1} u_{, x x}-a_{1} u_{, x}+\alpha_{1} u\left(t-\tau_{1}, x\right)+p_{1} u^{*} v, \\
& v_{, t} \leq d_{2} v_{, x x}-a_{2} v_{, x}+\alpha_{2} v\left(t-\tau_{2}, x\right) .
\end{aligned}
$$

We shall show that there exist $\lambda_{0}>0$ and $c_{0}>0$ such that

$$
(u, v)=\left(e^{-\lambda_{0}\left(x-c_{0} t\right)}, e^{-\lambda_{0}\left(x-c_{0} t\right)}\right)
$$

satisfies

$$
\begin{aligned}
& u_{, t} \geq d_{1} u_{, x x}-a_{1} u_{, x}+\alpha_{1} u\left(t-\tau_{1}, x\right)+p_{1} u^{*} v, \\
& v_{, t} \geq d_{2} v_{, x x}-a_{2} v_{, x}+\alpha_{2} v\left(t-\tau_{2}, x\right) .
\end{aligned}
$$

Substituting $(u, v)$ given by (3.15) into (3.16), we find that (3.16) is satisfied if and only if

$$
f\left(c_{0}, \lambda_{0}\right)>0, \quad g\left(c_{0}, \lambda_{0}\right)>0
$$

where

$$
\begin{aligned}
& f(c, \lambda):=c \lambda-d_{1} \lambda^{2}-a_{1} \lambda-\alpha_{1} e^{-\lambda c \tau_{1}}-p_{1} u^{*}, \\
& g(c, \lambda):=c \lambda-d_{2} \lambda^{2}-a_{2} \lambda-\alpha_{2} e^{-\lambda c \tau_{2}} .
\end{aligned}
$$

It is easily checked that for any fixed $\lambda>0$

$$
f_{, c}>0, \quad \lim _{c \rightarrow \infty} f(c, \lambda)=\infty, \quad \text { and } \quad g_{, c}>0, \quad \lim _{c \rightarrow \infty} g(c, \lambda)=\infty .
$$

Which indicate that there exist $\lambda_{0}>0$ and $0<c_{0}<\infty$ such that $f\left(c_{0}, \lambda_{0}\right)>0$ and $g\left(c_{0}, \lambda_{0}\right)>0$. Since $(u, v)=\left(e^{-\lambda_{0}\left(x-c_{0} t\right)}, e^{-\lambda_{0}\left(x-c_{0} t\right)}\right)$ satisfies (3.16) and 
solutions of (3.2) satisfy $(3.14),(u, v)=\left(e^{-\lambda_{0}\left(x-c_{0} t\right)}, e^{-\lambda_{0}\left(x-c_{0} t\right)}\right)$ is an upper solution for (3.2). Let $L_{1}$ be the time one solution map of the linear equation system corresponding to (3.16). We have that for $(u, v) \in \mathcal{C}_{\boldsymbol{\beta}}$, if $(u, v) \leq$ $\left(e^{-\lambda_{0} x}, e^{-\lambda_{0} x}\right)$ with $\theta \in[-\tau, 0]$ then

$$
T_{-c_{0}} Q_{1}[u, v](x) \leq T_{-c_{0}} L_{1}\left[\left(e^{-\lambda_{0}(\cdot)}, e^{-\lambda_{0}(\cdot)}\right)\right](x) \leq\left(e^{-\lambda_{0} x}, e^{-\lambda_{0} x}\right) .
$$

One can then use (2.5) to define the sequence $\mathbf{a}_{n}\left(c_{0} ; \theta, x\right)$ with $Q$ replaced by $Q_{1}$ and $(\phi(\theta, x), \psi(\theta, x))$ satisfying $(\phi(\theta, x), \psi(\theta, x)) \leq\left(e^{-\lambda_{0} x}, e^{-\lambda_{0} x}\right)$ for $\theta \in[-\tau, 0]$. Induction and (3.17) show that $\mathbf{a}_{n}\left(c_{0} ; \theta, x\right) \leq\left(e^{-\lambda_{0} x}, e^{-\lambda_{0} x}\right)$ for all $n$ and thus the limit function $\mathbf{a}\left(c_{0} ; \theta, x\right)$ satisfies $\mathbf{a}\left(c_{0} ; \theta, x\right) \leq\left(e^{-\lambda_{0} x}, e^{-\lambda_{0} x}\right)$. It follows immediately that

$$
\mathbf{a}\left(c_{0} ; \theta,+\infty\right)=\lim _{x \rightarrow \infty} \mathbf{a}\left(c_{0} ; \theta, x\right) \leq \lim _{x \rightarrow \infty}\left(e^{-\lambda_{0} x}, e^{-\lambda_{0} x}\right)=(0,0) .
$$

The definition of $c^{*}$ shows that $c_{0} \geq c^{*}$. This completes the proof of this lemma.

Proof of Theorem 3. By Theorem 2, we only need to show that for any $c \geq c^{*}$, there is no nonincreasing travelling wave solution in (3.2) that connects $\mathbf{0}=$ $(0,0)$ with $\boldsymbol{\nu}=\left(u^{*}, 0\right)$. Assume to the contrary that $(u(x-c t), v(x-c t))$ is a nonincreasing travelling wave solution in (3.2) with $c \geq c^{*}$ connecting $\mathbf{0}=(0,0)$ with $\boldsymbol{\nu}=\left(u^{*}, 0\right)$. Such a travelling wave solution is equivalent to a travelling wave solution for the following scalar equation:

$$
u_{, t}=d_{1} u_{, x x}-a_{1} u_{, x}-\alpha_{1}\left(u^{*}-u\left(t-\tau_{1}, x\right)\right)+\eta_{1}\left(u^{*}-u\right)^{2} .
$$

We therefore have $\hat{u}(x-c t)=u^{*}-u(x-c t)$ is a nondecreasing traveling wave solution of

$$
\hat{u}_{, t}=d_{1} \hat{u}_{, x x}-a_{1} \hat{u}_{, x}+\alpha_{1} \hat{u}\left(t-\tau_{1}, x\right)-\eta_{1} \hat{u}^{2}
$$

with $\hat{u}(-\infty)=0$ and $\hat{u}(+\infty)=u^{*}$. By Lemma $5, c^{*}>0$, so that $c>0$. Therefore, $\hat{u}(x-c t)$ is a nondecreasing traveling wave solution of (3.18) with the speed $-c<0$ connecting the unstable equilibrium 0 with the stable equilibrium $u^{*}$.

On the other hand

$$
\hat{u}_{, t} \leq d_{1} \hat{u}_{, x x}-a_{1} \hat{u}_{, x}+\alpha_{1} \hat{u}\left(t-\tau_{1}, x\right)
$$

which indicates that the time $t$ solution map of (3.18) is dominated by that of the linearized model. By Theorem 3.10 in Liang and Zhao [8] and the argument given on the pages 2758-2759 of Fang, Wei and Zhao [3] we obtain that the minimal traveling wave speed for (3.18) is given by $\inf _{\mu>0} \lambda(\mu) / \mu$ where $\lambda(\mu)$ is the principle eigenvalue of the characteristic equation

$$
\Delta(\lambda, \mu):=\lambda-d_{1} \mu^{2}-a_{1} \mu-\alpha_{1} e^{-\tau_{1} \lambda}=0 .
$$

Since $\lambda(0)>0$ and $\lambda(\mu) \geq d_{u} \mu^{2}$ for $\mu>0$, $\inf _{\mu>0} \lambda(\mu) / \mu>0$. We obtain a contradiction and the proof of this Theorem is complete. 


\section{Concluding Remarks}

In this paper we investigated a system of delayed reaction-diffusion equations which modelled growth, spread and competition of two species with stage structure in water flow. This model is an extension of the time-delayed population system with stage structure studied by Al-Omari and Gourley [2] and Zhang et al. [22]. There is no advection terms in their models, which can provide a resolution to the drift paradox in stream ecology (see [4]). "Drift paradox" is one key issue for theory in stream ecology and states that extinction is inevitable in a closed population subjected only to downstream drift. By using the abstract theorem in [7] we show that there exists a finite positive number $c^{*}$ that can be characterized as the slowest speed of travelling waves connecting two mono-culture equilibria or a mono-culture with the coexistence equilibrium. The existence result of travelling waves in [2] is improved.

In 2012, Li [6] showed that for the general partial cooperative reactiondiffusion system

$$
\mathbf{u}_{, t}=D \mathbf{u}_{, x x}-E \mathbf{u}_{, x}+\boldsymbol{f}(\mathbf{u}(t, x))
$$

$\bar{c}$ can be characterized as the slowest speed of a class of travelling wave solutions by verifying the linear determinacy conditions under appropriate assumptions, where

$$
\bar{c}:=\inf _{\mu>0} \frac{\gamma_{1}(\mu)}{\mu}
$$

and $\gamma_{1}(\mu)$ is the principal eigenvalue of the first irreducible block of the moment generating matrix $C_{\mu}$ of the linearized system of (4.1). However, in our system (1.7) the delay is considered in the vector function $\boldsymbol{f}(\mathbf{u}(t, x))$. Consequently the principal eigenvalue $\gamma_{1}(\mu)$ of $C_{\mu}$ and the corresponding eigenvector $\boldsymbol{\xi}(\mu)$ cannot be expressed explicitly. Thus the ideas used in [6] cannot be applied in our system.

In order to verify the linear determinacy conditions, one might first write the $\boldsymbol{\xi}(\mu)$ as a function of the principle eigenvalue $\gamma_{1}(\mu)$. Then use the fact that the wave speed equation $\phi(\mu)=\gamma_{1}(\mu) / \mu$ is a convex function and thus infimum in Eq. (4.2) exists, one might provide an estimation of $\gamma_{1}(\mu)$ and therefore show that the linear determinacy conditions are satisfied under appropriate assumptions. Liang and Zhao [8] developed the analytical theory on the spreading speeds for delayed cooperative system, which can be applied to our cooperative system (3.2). We shall study the issue on spreading speeds for model (1.7) in the following work.

\section{Acknowledgements}

The authors are grateful to the anonymous reviewers for his/her thoughtful comments and constructive suggestions on improving our original manuscript. This work is supported by Doctor Foundation of Northwest A\&F University (201104054460), NNSFC (39970112, 30470268, 11371294, and 11401474), and Research Fund for the Doctoral Program of Higher Education (20130204110004). 


\section{References}

[1] W.G. Aiello and H.I. Freedman. A time-delay model of single-species growth with stage structure. Math. Biosci., 101(2):139-153, 1990. http://dx.doi.org/10.1016/0025-5564(90)90019-U.

[2] J.F.M. Al-Omari and S.A. Gourley. Stability and traveling fronts in LotkaVolterra competition models with stage structure. SIAM J. Appl. Math., 63(6):2063-2086, 2003. http://dx.doi.org/10.1137/S0036139902416500.

[3] J. Fang, J. Wei and X.-Q. Zhao. Spatial dynamics of a nonlocal and time-delayed reaction-diffusion system. J. Differential Equations, 245(10):2749-2770, 2008. http://dx.doi.org/10.1016/j.jde.2008.09.001.

[4] A.E. Hershey, J. Pastor, B.J. Peterson and G.W. Kling. Stable isotopes resolve the drift paradox for Baetis mayflies in an arctic river. Ecology, pp. 2315-2325, 1993. http://dx.doi.org/10.2307/1939584.

[5] Y. Kuang and S.A. Gourley. Wavefronts and global stability in a time-delayed population model with stage structure. Proc. R. Soc. Lond. Ser. A Math. Phys. Eng. Sci., 459(2034):1563-1579, 2003.

[6] B. Li. Traveling wave solutions in partially degenerate cooperative reactiondiffusion systems. J. Differential Equations, 252(9):4842-4861, 2012. http://dx.doi.org/10.1016/j.jde.2012.01.018.

[7] B. Li and L. Zhang. Travelling wave solutions in delayed cooperative systems. Nonlinearity, 24(6):1759, 2011. http://dx.doi.org/10.1088/0951$7715 / 24 / 6 / 004$.

[8] X. Liang and X.-Q. Zhao. Asymptotic speeds of spread and traveling waves for monotone semiflows with applications. Comm. Pure Appl. Math., 60(1):1-40, 2007. http://dx.doi.org/10.1002/cpa.20154.

[9] R. Lui. Biological growth and spread modeled by systems of recursions. II. Biological theory. Math. Biosci., 93(2):297-311, 1989.

[10] G. Lv and M. Wang. Traveling wave front in diffusive and competitive LotkaVolterra system with delays. Nonlinear Anal. Real World Appl., 11(3):13231329, 2010. http://dx.doi.org/10.1016/j.nonrwa.2009.02.020.

[11] R.H. Martin and H.L. Smith. Abstract functional-differential equations and reaction-diffusion systems. Trans. Amer. Math. Soc., 321(1):1-44, 1990.

[12] J.D. Murray. Mathematical Biology I: An Introduction. Springer, 2002.

[13] J.D. Murray. Mathematical Biology I: II: Spatial Models and Biomedical Applications. Springer, 2002.

[14] A. Okubo and S.A. Levin. Diffusion and Ecological Problems: Modern Perspectives, volume 14 of Interdiscip. Appl. Math. Springer, 2001.

[15] N. Shigesada and K. Kawasaki. Biological Invasions: Theory and Practice. Oxford University Press, 1997.

[16] D. Tilman and P.M. Kareiva. Spatial Ecology: The Role of Space in Population Dynamics and Interspecific Interactions, volume 30. Princeton University Press, 1997.

[17] N.K. Vitanov, Z.I. Dimitrova and K.N. Vitanov. Traveling waves and statistical distributions connected to systems of interacting populations. Comput. Math. Appl., 66(9):1666-1684, 2013. http://dx.doi.org/10.1016/j.camwa.2013.04.002. 
[18] H. Wang. On the existence of traveling waves for delayed reaction-diffusion equations. J. Differential Equations, 247(3):887-905, 2009.

http://dx.doi.org/10.1016/j.jde.2009.04.002.

[19] H.F. Weinberger, M.A. Lewis and B. Li. Analysis of linear determinacy for spread in cooperative models. J. Math. Biol., 45(3):183-218, 2002.

http://dx.doi.org/10.1007/s002850200145.

[20] J. Wu and X. Zou. Traveling wave fronts of reaction-diffusion systems with delay. J. Dynam. Differential Equations, 13(3):651-687, 2001.

http://dx.doi.org/10.1023/A:1016690424892.

[21] L. Zhang and B. Li. Traveling wave solutions in an integro-differential competition model. Discrete Contin. Dyn. Syst. Ser. B, 17(1):417-428, 2012. http://dx.doi.org/10.3934/dcdsb.2012.17.417.

[22] L. Zhang, B. Li and J. Shang. Stability and travelling waves for a time-delayed population system with stage structure. Nonlinear Anal. Real World Appl., 13(3):1429-1440, 2012. http://dx.doi.org/10.1016/j.nonrwa.2011.11.007. 